\title{
Using semantics for representing experimental protocols
}

\author{
Olga Giraldo ${ }^{\text {* }}$ (D), Alexander García ${ }^{1}$, Federico López² and Oscar Corcho
}

\begin{abstract}
Background: An experimental protocol is a sequence of tasks and operations executed to perform experimental research in biological and biomedical areas, e.g. biology, genetics, immunology, neurosciences, virology. Protocols often include references to equipment, reagents, descriptions of critical steps, troubleshooting and tips, as well as any other information that researchers deem important for facilitating the reusability of the protocol. Although experimental protocols are central to reproducibility, the descriptions are often cursory. There is the need for a unified framework with respect to the syntactic structure and the semantics for representing experimental protocols.

Results: In this paper we present "SMART Protocols ontology", an ontology for representing experimental protocols. Our ontology represents the protocol as a workflow with domain specific knowledge embedded within a document. We also present the $\boldsymbol{S}$ ample Instrument $\boldsymbol{R}$ eagent $\mathbf{O}$ bjective (SIRO) model, which represents the minimal common information shared across experimental protocols. SIRO was conceived in the same realm as the Patient Intervention Comparison Outcome (PICO) model that supports search, retrieval and classification purposes in evidence based medicine. We evaluate our approach against a set of competency questions modeled as SPARQL queries and processed against a set of published and unpublished protocols modeled with the SP Ontology and the SIRO model. Our approach makes it possible to answer queries such as Which protocols use tumor tissue as a sample.

Conclusion: Improving reporting structures for experimental protocols requires collective efforts from authors, peer reviewers, editors and funding bodies. The SP Ontology is a contribution towards this goal. We build upon previous experiences and bringing together the view of researchers managing protocols in their laboratory work. Website: https://smartprotocols.github.io/.
\end{abstract}

Keywords: Semantic web, Graph theory, Ontologies, RDF for experimental protocols, Knowledge representation, Linked data

\section{Background}

Experimental protocols are fundamental information structures that support the description of the processes by means of which results are generated in experimental research [1]. Experimental protocols describe how the data were produced, the steps undertaken and conditions under which these steps were carried out. Biomedical experiments often rely on sophisticated laboratory protocols, comprising hundreds of individual steps; for instance, the protocol for chromatin immunoprecipitation on a microarray (Chip-chip) has 90 steps and uses over 30 reagents and 10 different devices [2]. Nowadays, such

\footnotetext{
*Correspondence: ogiraldo@fi.upm.es

'Ontology Engineering Group, Madrid, Universidad Politécnica de Madrid,

28660 Madrid, Spain

Full list of author information is available at the end of the article
}

protocols are generally written in natural language and presented in a "recipe" style, so as to make it possible for researchers to reproduce the experiments.

The quality of experimental protocols reported in articles is a cause of concern. Reproducibility, central to research, depends on well-structured and accurately described protocols. Kilkenny et al. [3] found that 4 percent of the 271 journal articles assessed did not report the number of animals used anywhere in the methods or the results sections. Assessing statistical significance requires to know the number of animals participating in an experiment; it is also necessary if the experimental methods are to be reproducible, reused and adapted to similar settings. High-quality description of experimental methods is also critical when comparing results and integrating data. In 
an effort to address the problem of inadequate methodological reporting, journals such as Nature Protocols [4], Plant Methods (Methodology) [5] and Cold Spring Harbor Protocols [6], have guidelines for authors that include recommendations about the information that should be documented in the protocols. The ISA-TAB also illustrates work in this area; it delivers metadata standards to facilitate data collection, management and reuse from "omic-based" experiments [7]. The BRIDG initiative [8] aims to formalize a shared view of the dynamic and static semantics of protocol-driven research. The BioSharing initiative [9], is a catalog of standards promoting the representation of information in the life, environmental and biomedical sciences [9]. STAR [10] is an effort that proposes to "Empowering Methods" offering a overview of resources used in a study. Ontologies such as EXACT $[11,12]$ aim to formalize the description of protocols focusing on experimental actions; the BioAssay Ontology (BAO) [13] describes biological screening assays and their results; the eagle-i resource ontology (ERO) [14] represents some aspects related to protocols.

Here we present SMART Protocols ontology (henceforth SP), our ontology for representing experimental protocols; we aim to "facilitate the semantic representation of experimental protocols". Our representation makes it possible to answer queries such as "Which protocols use "tumor tissue" as a sample?", "Retrieve the reagents and the corresponding information from the manufacturers for a specific protocol", "retrieve the diseases caused by the reagents used in a specific protocol". These and other queries can be processed at our SPARQL endpoint ${ }^{1}$. The SP Ontology provides the structure and semantics for data elements common across experimental protocols. For representing reagents, samples, instruments and experimental actions we reuse ontologies such as the Chemical Entities of Biological Interest (ChEBI) [15], NCBI taxonomy [16-18], the Ontology for Biomedical Investigations (OBI) [19], the BioAssay Ontology (BAO), The Experimental Factor Ontology (EFO) [20], eaglei resource ontology (ERO), Cell Line Ontology (CLO) $[21,22]$, and EXACT. We also reuse and extend classes from the Information Artifact Ontology (IAO) [23]. In this paper we also present the SIRO model; this is a minimal information model for the representation of Samples Instruments Reagents Objective (hence SIRO). This model has been conceived in a way similar to that of the Patient Intervention Comparison Outcome (PICO) model; it helps to frame questions and provides an anchor for the records [24]. SIRO facilitates classification and retrieval without exposing the content of the document. In this way, publishers and laboratories may keep the content private, exposing only the information that describes the sample, instruments, reagent and objective of the protocol. As an illustration, in this paper we use the protocol
"Extraction of total RNA from fresh/frozen tissue (FT)" [25] as a running example. We represent this protocol with the SP ontology and SIRO.

\section{Methods}

Our SMART Protocols ontology [26] is based on an exhaustive analysis of 175 published and unpublished experimental protocols (see Table 1 in Domain Analysis and Knowledge Acquisition, DAKA); we also analyzed on-line repositories and guidelines for authors. For the development of the SP Ontology [1] we have followed the practices recommended by the NeOn methodology [27], as well as those reported by García [28]. For example, we used conceptual maps to better understand the correspondences, relations and possible hierarchies in the knowledge we were representing. The stages and activities we implemented throughout our ontology development process are illustrated in Fig. 1 and explained below. For the ontology development process we also considered the guidelines from the OBO foundry [29].

\section{The kick-off, scenarios and competency questions}

In the first stage, we gathered motivating scenarios, competency questions, and requirements. We focused on the functional aspects that we wanted the ontology to represent. Domain experts were asked to provide us with a list of competency questions, these are presented in our website $^{2}$. Some of the competency questions we gathered include, "retrieve the protocols using a given sample" and, "which protocols can I use to process this sample given that I only have $\mathrm{X}$ and $\mathrm{Z}$ reagents". Competency questions were initially used to scope the domain for which we were developing the ontology; these questions were also used during the evaluation.

\section{Conceptualization and formalization}

In this stage we identified reusable terminology from other ontologies; for supporting activities throughout this stage we used BioPortal [30] and Ontobee [31]. We also looked into minimal information standards [32], guidelines and vocabularies representing research activities [33-35]. Issues about axioms required to represent this domain were discussed and tested in Protégé v. 4.3 and 5.0 [36]; during the iterative ontology building, classes and properties were constantly changing. We identified, and explain below, three main activities throughout this stage,

Table 1 Repositories and number of protocols analyzed

\begin{tabular}{lllllllllll}
\hline Repository & Bio Tech & CSH & CP & GMR & JoVE & NPE & PM & PO & SP & CIAT \\
\hline No. of protocols & 6 & 9 & 25 & 5 & 21 & 13 & 12 & 5 & 4 & 75 \\
Total & & & & & 175 & & & & & \\
\hline
\end{tabular}

The protocols are available at: https://smartprotocols.github.io/ 


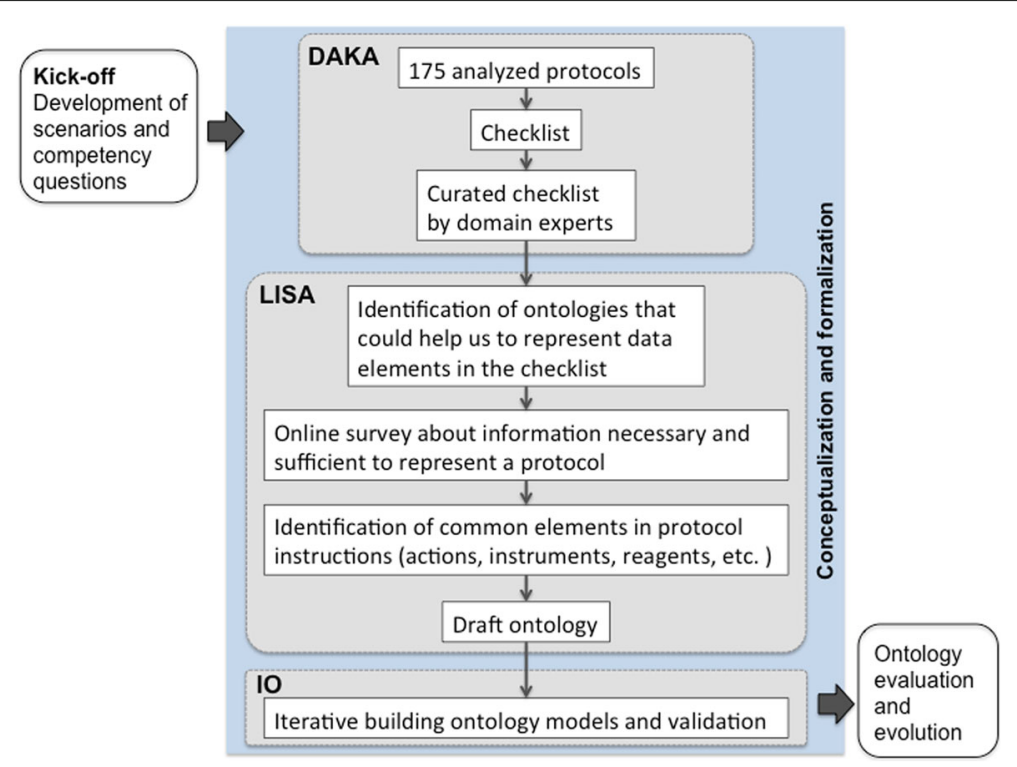

Fig. 1 Developing the SMART Protocols ontology, methodology

namely: Domain Analysis and Knowledge Acquisition (DAKA), Linguistic and Semantic Analysis (LISA), Iterative ontology building and validation (IO).

\section{Domain analysis and knowledge acquisition, DAKA}

We manually reviewed 175 published and unpublished protocols from topic areas such as molecular biology, cell and developmental biology, biochemistry, biotechnology, microbiology and virology, as well as guidelines for authors from journals. The unpublished protocols (75 in total) were collected from four laboratories located at The International Center for Tropical Agriculture (CIAT) [37]. The published protocols (open access) were gathered from 9 repositories; Table 1 presents the list of journals and the number of protocols that we analyzed. We used these sources to prepare a checklist with data elements that were required in guidelines for authors and also present in published protocols -see Annex $1^{3}$. This was the seed for our discussions with domain experts.

Our domain analysis focused on gathering terminology and data elements, idem higher abstractions that could be used to group terminology. Domain experts were bringing their protocols and discussing specific issues, e.g. what was missing for applying a particular protocol. As the discussions were progressing, published and unpublished protocols were added to the mix. Due to time constraints domain experts were not required to work before or after the workshops. Olga Giraldo was the facilitator for the DAKA activities. This made the processes with domain experts more efficient because she has extensive experience in laboratory practices. Ten domain experts participated in DAKA; they all had hands-on experience in areas such as molecular biology, virology, plant breeding, biochemistry, clinical microbiology and pathology. From DAKA we confirmed most of the data elements in our initial checklist and identified clusters of terminology, e.g. samples and instruments. The output of this activity was an improved checklist and relations to the information in the protocols. This output was used as input for the linguistic analysis.

\section{Linguistic and semantic analysis, LISA}

From our corpus of protocols we selected 100 documents; these represented the topic areas for which we had domain experts. We tried to have some complex and lengthy protocols involving several procedures and technologies; for instance, protocols describing the development of an SNP genotyping resource [38] and protocols describing the construction of an RNA-seq library [39]. We also worked with simpler protocols such as sample preparation or DNA extraction protocols. The terminology gathered in DAKA was discussed with domain experts and analyzed against existing ontologies; BioPortal and Ontobee were used to browse the ontologies in order to determine how terms were related to biomedical ontologies and which were the ontologies that could be relevant for this work.

Throughout this activity we also addressed the representation of workflows in the protocols. This was particularly problematic because domain experts did not agree on how granular the descriptions of the workflows and the relation between steps needed to be, how to indicate order in the sequence of operations and, what information was obligatory in the description of the steps. In this activity we used an on-line survey that helped us to 
determine and validate what data elements were necessary and sufficient for the description of the protocols -see Annex $2^{3}$. We used the outputs from DAKA in the survey and asked participants to indicate whether a particular data element was relevant or not; an invitation to participate was circulated over mailing lists, participants did not have to disclose their identity. Twenty participants filled up the survey; this survey helped us to informally validate the outputs from DAKA and also gave us another perspective about relevant data elements in the description of protocols. Results from the survey are available in Annex $3^{3}$.

From this activity, we identified linguistic structures that authors were using to represent actions. We were interested in understanding how verbs were representing actions and what additional information was indicating the attributes for actions. For instance, "Fresh-leaf tissue $(0.2 \mathrm{~g})$ was ground in a 1.5-mL Eppendorf tube with a micropestle and preheated freshly prepared $800 \mathrm{uL}$ extraction buffer was immediately added to the tube" [40] is a commonly used cell disruption step in nucleic acids and protein extraction protocols. In our corpus of documents, these steps were usually described using verbs like "break, chop, grind, homogenize". There are also common methods for specific operations; for instance, for breaking the cells the methods were "blending, grinding or sonicating" the sample. The sequence of instructions had an implicit order that was not always clearly specified as authors sometimes hide it in the narrative. There is, however, an input-output structure. Actions in the workflow of instructions are usually indicated by verbs; accurate information for implementing the action implicit in the verb was not always available. For instance, structures such as "Mix thoroughly at room temperature", "Briefly spin the racked tubes" are common in our dataset. The instructions always have actions and participants, which may be samples, reagents, instruments and/or measures. This was particularly useful in the definition of our workflow; the pattern that emerged is discussed in the "Results" section. In this activity we also identified document-related data elements; for instance, roles for authors, e.g. validator, statistical reviewer. We also identified the ontologies that could represent the concepts we were working with. A draft ontology with the seminal terminology and initial classification was the output from LISA; this output was further refined during the iterative ontology building stage.

\section{Iterative ontology building and validation, 10}

The draft ontology from LISA was incrementally growing in complexity, number of concepts and relations. The knowledge engineer conducted continuous evaluations of the draft ontologies against competency questions. The ontology models were shared with domain experts, they reviewed the drafts, gave feedback and the ontology was updated.

As we were building ontology models, we identified the modularity needed to represent experimental protocols. From our models, we conceptualized the protocols as workflows embedded within documents. Thus, the document module of SP ontology (henceforth SP-Document) was designed to provide a structured vocabulary that could represent information for reporting an experimental protocol. The workflow module of SP ontology (henceforth SP-workflow) delivers a structured vocabulary to represent the sequence of actions in the execution of experimental protocols. The main outcome from this activity was an ontology with the SP-Document and SPworkflow modules and their corresponding classes and object properties. Our ontologies were developed using OWL-DL. We used the Protégé editor versions 4.X and 5; the Protégé plug-in OWLViz [41] was used to visualize the model.

\section{Ontology evaluation}

during the evaluation process, we addressed issues related to the syntax, the conceptualization and formalization. We also verified whether the competency questions could be resolved by representing experimental protocols using the ontology and having the resulting RDF in a SPARQL endpoint.

We evaluated the syntax of the ontology using The OntOlogy Pitfall Scanner (OOPS) [42]; it was useful to detect and correct anomalies or pitfalls in our ontologies [43]. For instance, the identification of incomplete inverse object properties, lack of domain and range, missing annotations and issues in naming conventions. The resulting ontology from the "Conceptualization and Formalization" phase was evaluated by 10 domain experts. They were asked to determine if the proposed classes in the ontology could represent the information from a set of 13 protocols that we selected for this purpose. A list of the protocols as well as results from this evaluation are presented in Annex $4^{3}$.

We also tested the capability of the SMART Protocols ontology to answer the competency questions specified by domain experts; does the ontology represent enough information to answer these types of questions? do the answers require a particular level of detail or representation of a particular area? This part of the evaluation entailed the transformation of 10 experimental protocols to $\mathrm{RDF}^{4}$. These were uploaded in our SPARQL endpoint and the queries were formalized in SPARQL; a complete list of SPARQL queries has been made available ${ }^{2}$.

\section{Results}

\section{The SMART protocols ontology}

Our ontology reuses BFO; we are also reusing the ontology of relations ( $\mathrm{RO})$ [44] to characterize concepts. In 
addition, each term in the SP ontology is represented with annotation properties imported from OBI Minimal metadata [45]. The classes, properties and individuals are represented by their respective labels to facilitate readability. The prefix indicates the provenance for each term; for instance, the prefix sp is used to identify classes and object properties from SP ontology. For the object properties we are using italics, words or phrases representing instances are in between quotation marks, e.g. "RNA extraction", instance of the class sp:lab procedure 3. In this section we use the protocol "Extraction of total RNA from fresh/frozen tissue (FT)" [25] as a running example to represents the document and workflow aspects of a protocol. Our ontology is available in BioPortal ${ }^{5}$, github ${ }^{6}$ and also is registered at vocab.linkeddata.es ${ }^{7}$. vocab.linkeddata.es is a list of vocabularies developed by the Ontology Engineering Group (OEG). A graphical illustration of the ontology can be found at Annex $5^{3}$.

\section{The document module}

The document module of the SP ontology [46] aims to provide a structured vocabulary of terms to represent information for reporting an experimental protocol. The class iao:information content entity and its subclasses iao:document, iao:document part, iao:textual entity and iao:data set were imported from IAO. This module represents metadata elements as classes, some of them are: sp:title of the protocol, sp:purpose of the protocol, sp:application of the protocol, sp:reagent list, sp:equipment and supplies list, $\mathrm{sp}$ :manufacturer, sp:catalog number and sp: storage conditions. We have used the SPDocument modeule to represent our running example, the results are presented in Table 2 and Fig. 2; metadata elements are organized in SP-Document as information content entities. In order to facilitate the use of identifiers for the material entities like reagents and equipments, we created the object property $s p$ has catalog number and the class $\mathrm{sp}$ :catalog number. In this way a relation is established between the reagent or equipment and the corresponding manufacturer.

\section{The workflow module}

The SP ontology also considers the protocol as an executable element to be carried out and maintained by humans. The workflow module [47] is a descriptive model for workflows; it is not a workflow programming language. The workflow module represents the procedures, subprocedures, actions (or verbs), experimental inputs (samples/specimens) and other participants such as reagents and instruments. Experimental protocols often include a set of laboratory procedures; these transform inputs into outputs. Our running example (see
Fig. 3 and Table 3), includes 3 laboratory procedures: $\mathrm{sp}$ : lab procedure 1 ("Protocol overview", indicating how to process the sample), sp:lab procedure 2 ("Prior to RNA extraction: cleaning process of equipment") and sp: lab procedure 3 ("RNA extraction"). The first column in Table 3 includes the procedures from our running example. The second column includes subprocedures or instructions for each procedure.

The class sp:lab procedure 1 ("Protocol overview") has a tumor tissue (nci:tumor tissue) as an input (sp:has experimental input); in a similar way, the lab procedure 1 has a homogenized tissue (sp:homogenized tissue) as an output (sp:has output). The laboratory procedure 1 includes 3 subprocedures (or steps/instructions) indicating how to manipulate and prepare the sample, namely: $\mathrm{sp}: 1 \mathrm{ab}$ subprocedure 1.1, sp:lab subprocedure 1.2 and sp:lab subprocedure 1.3. The order in which these subprocedures should be executed is represented by the BFO property is preceded by and precedes. The class $\mathrm{sp}$ : lab procedure 2 ("Prior to RNA extraction: cleaning process of equipment") is a recipe describing how to clean the equipment to be used during the RNA extraction protocol. This recipe includes 3 steps, $\mathrm{sp}: 1 \mathrm{ab}$ subprocedure2.1, sp:lab subprocedure 2.2 and sp:lab subprocedure 2.3 .

The class sp: lab procedure 3 ("RNA extraction") has the homogenized tissue (output from the lab procedure 1) as an input and, the class chebi:RNA as an output. It includes 20 subprocedures, these are not represented in the Fig. 3 due to lack of space. We propose the classes $\mathrm{sp}$ : laboratory procedure and sp:laboratory subprocedure for the representation of procedures and subprocedures. The object property, sp: has procedure, is used to characterize the laboratory procedures that are part of the execution of an experimental protocol (sp:experimental protocol execution); the object property sp:has subprocedure, is used to characterize the subprocedures that are part of a given procedure. Procedures have inputs and outputs, subprocedures have participants. For cases where authors only have an extensive list of steps, the SP ontology considers these as subprocedures under a procedure container. In this way we are representing protocols with only a long list of steps as well as those with groups of steps. This also allows us to represent more complex protocols that usually result from merging several protocols.

We are representing antibodies, cell lines and plasmids as material entities. We are using ro:derives from to indicate that it derives from an organism; similarly, we are using the obi:has role to indicate the role that it plays, as understood by the author of the protocol. 
Table 2 Metadata represented in SP-Document

\begin{tabular}{|c|c|}
\hline \multicolumn{2}{|l|}{ Bibliographic metadata } \\
\hline sp:title of the protocol & Extraction of total RNA from fresh/frozen tissue (FT) \\
\hline sp:author name & $\begin{array}{l}\text { "Kim M. Linton", "Yvonne Hey", "Sian Dibben", "Crispin J. Miller", "Anthony J. Freemont", "John A. Radford", } \\
\text { and "Stuart D. Pepper" }\end{array}$ \\
\hline sp:protocol identifier & DOl:10.2144/000113260 \\
\hline \multicolumn{2}{|l|}{ Descriptive metadata } \\
\hline sp:application of the protocol & $\begin{array}{l}\text { "Methods comparison for high-resolution transcriptional analysis of archival material on Affymetrix Plus } 2.0 \text { and Exon } \\
1.0 \text { microarrays" }\end{array}$ \\
\hline sp:provenance of the protocol & "The extraction method (steps 2-21) is taken from the method supplied with TRIzol reagent Invitrogen, Paisley, UK)." \\
\hline \multicolumn{2}{|l|}{ Metadata about the materials } \\
\hline sp:specimen name & "tumor tissue" \\
\hline sp:reagent name & "TRIzol", "Chloroform”, “Ethyl alcohol”, "Isopropyl alcohol” \\
\hline sp:manufacturer name & "Invitrogen", "Sigma-Aldrich" \\
\hline sp:equipment or supplies name & "Tissue storage container", "Homogenizer blades", "Forceps", "Scalpel”, "Scalpel holder" \\
\hline
\end{tabular}

Evaluation

Syntax

OOPS allowed us to identify the lack of domain and range in the object properties ro:part_of and ro:has_part; these were imported from the Relations Ontology (RO). We verified in the original ontology and these two properties do not have domain and range [48]. OOPS was useful for verifying the syntax of the ontology.

\section{Conceptualization and formalization}

The resulting ontology was evaluated by 10 domain experts, they were asked to determine whether the resulting ontology was representing the information items from experimental protocols. This evaluation was satisfactory because the information from the protocols was represented in the ontology. Interestingly, resulting from this evaluation we could identify some issues related to the way published and unpublished protocols were described. For instance, published protocols don't have any information that facilitates the identification of roles; for instance, who is the chief scientist, who did the statistical validation, who was the lab scientist, etc. Identifying these roles was considered as important because it is an indication of quality control in the development of the protocol; this data element was identifiable in unpublished protocols and it is part of our ontology. Unpublished protocols usually have version information, as well as a short description of the roles played by those who are using, developing, standardizing or modifying the protocols.

From this evaluation it was also evident that published protocols were not consistent in the data elements that they use to represent the experimental protocol. For instance, some of the protocols had an explicit description of "advantages" and "application of the protocol", while some others did not provide this information. A similar situation was found with respect to information about limitations. The bibliographic metadata that was identified includes, title, author, subject area and protocols identifiers (IDs). These were not always available; in the case of unpublished protocols the ID was sometimes an internal code. Although the class author identifier (sp:author identifier) could not be instantiated, we decided to leave it in the ontology because it was deemed important. Published and unpublished protocols have authors as literal values without any relation to IDs.

Published and unpublished protocols often report the name of the materials but not the manufacturer and the corresponding identifier, this is usually the catalog number. This information is frequently available and it is always necessary when trying to reuse a protocol, the SP Ontology models these data elements. Alert messages, hints, pause points, cautions or troubleshooting were represented in SMART Protocols ontology and validated by the domain experts. Although the description of the work steps, procedures, subprocedures and recipes varied across the protocols, the data elements describing the workflow could be easily represented in our ontology.

We also asked domain experts to instantiate the classes with text from the protocols. They were selecting excerpts of text and assigning classes to these narratives, e.g. "This is a simple protocol for isolating genomic DNA from fresh plant tissues" was classified as an objective, "DNA from this experiment can be used for all kinds of genetics 


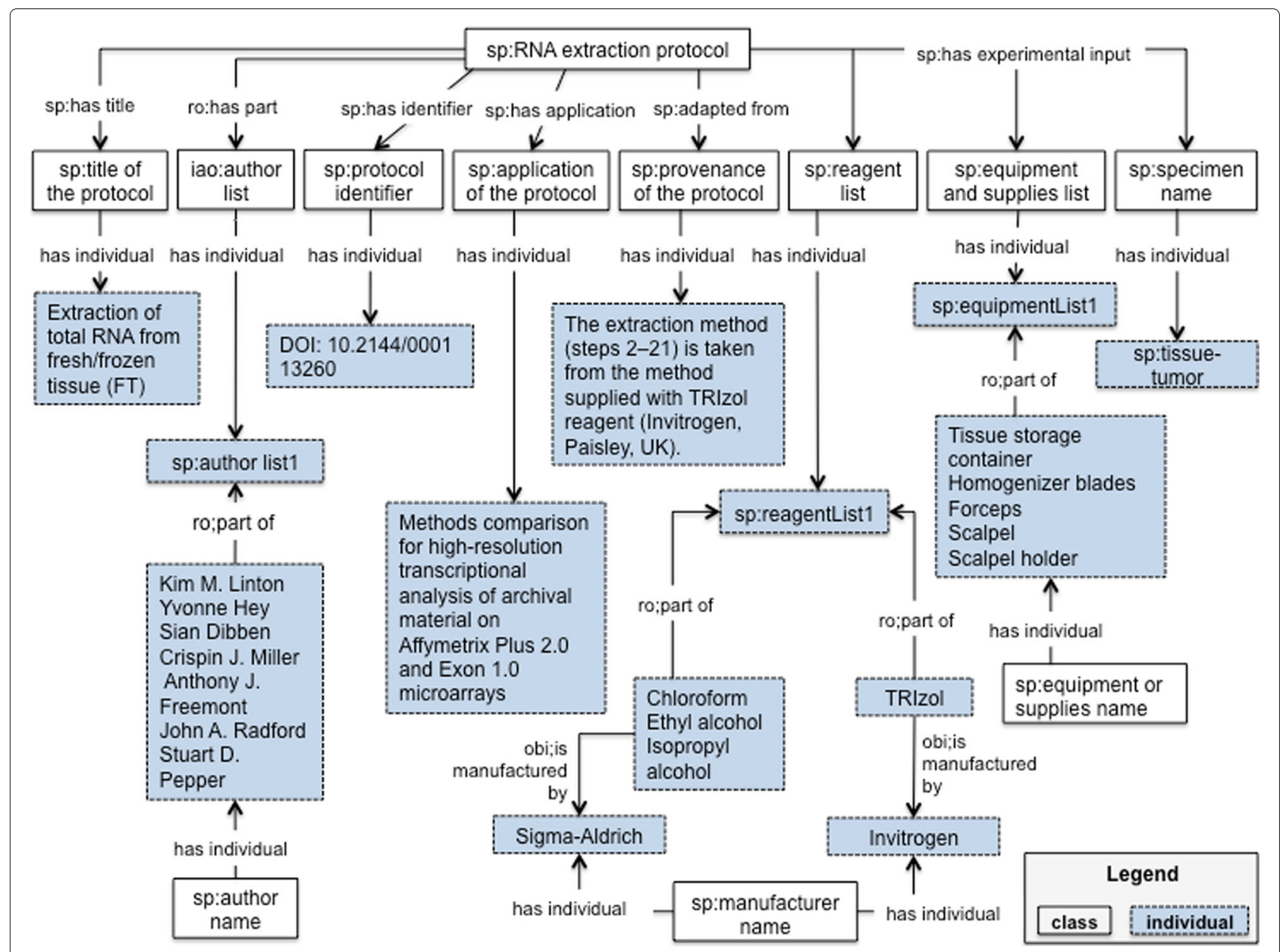

Fig. 2 SP-Document module. This diagram illustrates the metadata elements described in Table 2. The classes, properties and individuals are represented by their respective labels

studies, including genotyping and mapping" was classified as an application. They were also selecting some specific words and classifying them; for instance, "Isopropanol" was classified as a reagent, "mortar and pestle" was classified as an equipment. Information related to the overall objective of the protocol, applications, advantages, limitations and provenance was represented in our ontology; these data elements were validated by domain experts as they were mapping them to the ontology. Information about the sample (strain, line, genotype, developmental stage, organism part, growth conditions, treatment type and quantity used) was identified in published and unpublished protocols and could easily be mapped to the ontology.

Materials were also identified and mapped; interestingly, domain experts recognized different types of materials, for instance, instruments (including laboratory consumables), reagents, kits and software. In the resulting ontology we included "reagent" and "kit" under material entities; this made it easier for domain experts to identify terminology related to these classes. Published and unpublished protocols don't differentiate across reagents, recipes, and kits; these are all usually listed under "Reagents". However, domain experts reusing the protocols understand these under different categories. Reagents are understood as "ready to use", often purchased; they also included mixtures prepared in the lab under reagents. Reagents are substances used in a chemical reaction to detect, measure, examine, or produce other substances [49]. Kits were considered as "gear consisting of a set of articles or tools for a specified purpose". For instance, the Qiagen RNeasy Spin mini is a kit for purification of RNA from cells and tissues. However, a kit could also be an instrument; for instance, a digital recording transcribing kit, an instrument used to digitally record speech for transcription.

Recipes were identified as the most appropriate part of the protocol for including the details indicating how 


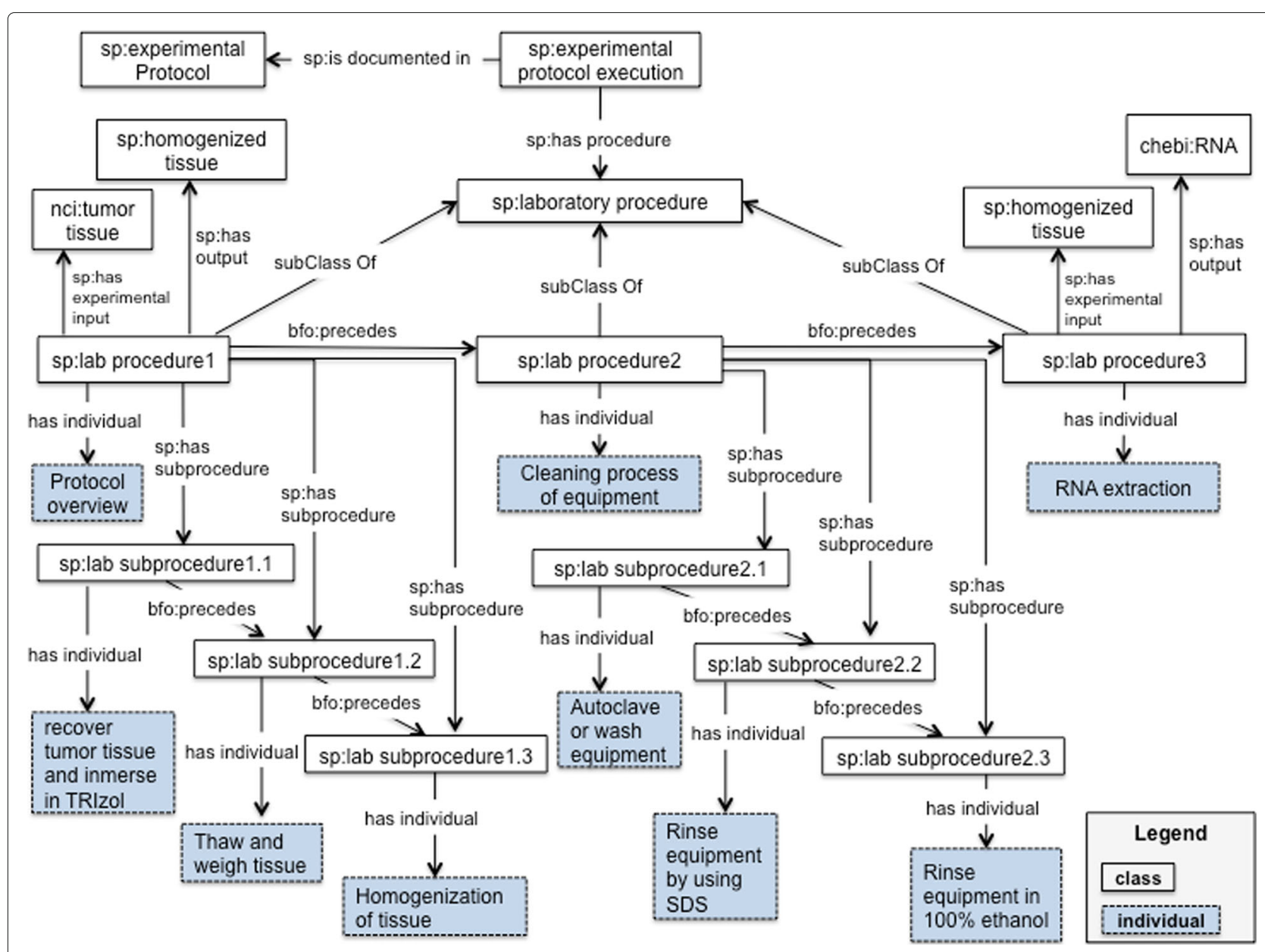

Fig. 3 SP-Workflow module. This diagram illustrates the metadata elements described in Table 3. The classes, properties and individuals are represented by their respective labels

to prepare a particular solution, media, buffer, etc. The recipes could also describe how to make something; for example, "recipes describing how to clean laboratory equipment before starting the execution of a procedure", see lab procedure 2 in our running example (Fig. 3 and Table 3); a recipe also is a way to include details regarding, e.g., the setup of HPLC separation methods. We classified the term "recipe" as a textual entity. The execution of a recipe was also considered, we included the term "recipe execution" as a planned process.

\section{Competency questions}

The RDF generated from instantiating the ontology was loaded in our SPARQL endpoint; the competency questions were then executed against this dataset. In general the expected information was retrieved; however, as domain experts were looking at the results, they started to reformulate the questions by asking for more information.
For instance, domain experts asked for reagents to be linked to catalogs from the manufacturers or to resources like PubChem [50]. They were also interested in linking the samples/organisms to DBPEDIA [51] and NCBI taxonomy database [17, 18]; similarly, safety information was deemed as another case for establishing links between entities in the protocol and other information resources in the web. Some queries making use of linked data resources via federated queries illustrate this requirement; as additional information was necessary, we were looking into linked data resources that could complement the retrieved information. Queries like "Retrieve all the reagents and the information about where to buy them" illustrate how we were making use of other information resources; federated queries, $\mathrm{see}^{2}$, are retrieving complementary information from linked data resources such as DBpedia, Uniprot [52], PubChem, SNOMED over BioPortal and ChEBI. Some of the federated queries are presented in Table 4. 
Table 3 Procedures and subprocedures from "Extraction of total RNA from fresh/frozen tissue (FT)"

\begin{tabular}{|c|c|}
\hline Procedure & Subprocedure \\
\hline $\begin{array}{l}\text { Protocol overview (sp:lab } \\
\text { procedure 1) }\end{array}$ & $\begin{array}{l}\text { Recover tumor tissue at the time of } \\
\text { surgery, trim into } 1 \text {-cm } 3 \text { fragments, } \\
\text { and immerse immediately in TRIzol } \\
\text { reagent prior to freezing at }-80^{\circ} \text {. } \\
\text { Thaw and weigh tissue prior to RNA } \\
\text { extraction, working quickly. } \\
\text { Use a tissue power homogenizer (or } \\
\text { a mortar and pestle) to homogenize } \\
\text { tissue by hand. }\end{array}$ \\
\hline $\begin{array}{l}\text { Prior to RNA extraction: cleaning } \\
\text { process of equipment (sp:lab proce- } \\
\text { dure 2) }\end{array}$ & $\begin{array}{l}\text { Autoclave or wash equipment (i.e., } \\
\text { tissue storage container, homoge- } \\
\text { nizer blades, forceps, scalpel holder) } \\
\text { in Neutracon solution for } 2-4 \mathrm{~h} \text {. } \\
\text { Rinse equipment well in } 1 \% \text { SDS } \\
\text { (prepared using DEPC-treated or } \\
\text { other nuclease-free water). } \\
\text { Rinse in } 100 \% \text { ethanol and leave to } \\
\text { air-dry. }\end{array}$ \\
\hline RNA extraction (sp:lab procedure 3) & $\begin{array}{l}\text { Homogenize sample using tissue } \\
\text { homogenizer. } \\
\text { Add } 0.2 \mathrm{~mL} \text { chloroform per } 1 \mathrm{~mL} \\
\text { TRIzol and cap tube tightly. } \\
\text { Add } 0.5 \mathrm{~mL} \text { isopropyl alcohol per } 1 \\
\mathrm{~mL} \text { TRIzol. } \\
\text { Add } 1 \mathrm{~mL} 75 \% \text { ethanol per } 1 \mathrm{~mL} \\
\text { TRIzol and vortex for } 10 \mathrm{~s} \text {. }\end{array}$ \\
\hline
\end{tabular}

\section{Applying the SMART protocols ontology to the definition of a minimal information model}

Initially we developed the SP ontology and then the SIRO model. As we were representing the protocols as RDF we were also analyzing the competency questions; by doing so we saw a common pattern. From our competency questions, 17.4 percent were related to Samples, 8.7 percent were related to Instruments, 34.8 percent were related to Reagents Fig. 4. Furthermore, although the description of the workflow varies across our evaluation corpus, these data elements were always present. We focused on the manual identification of commonalities, the very minimal information shared across our corpus of documents. We then classified these data elements by mapping them to the SP ontology. This allowed us to determine higher abstractions to which the terminology could be mapped, e.g., "sample", "reagent" and "instrument". Domain experts discussed the granularity of the workflow description, whether the limitations of the protocol should or should not be reported, how to report the application of the protocol, etc. However, there was no disagreement about the need to report the objective of the protocol, e.g. "method for the production of $3 D$ cell lysates that does not compromise cell adhesion before cell lysis". Unlike samples, instruments and reagents, the objective is not always easily identifiable; it may be scattered throughout the document. It is, however, an important element; the description of the objective makes it easier for the readers to decide on the suitability of the protocol for their experimental problem. The SIRO model is illustrated in Fig. 5.

\section{The sample instrument reagent objective (SIRO) model}

SIRO represents the minimal common information shared across experimental protocols. It serves two purposes. First, it extends available metadata for experimental protocols, e.g. author, title, date, journal, abstract, and other properties that are available for published experimental protocols. SIRO extends this layer of metadata by aggregating information about Sample, Instrument, Reagent and Objective -hence the name. Categories and instances of the data elements for SIRO are presented in Table 5. Second, SIRO makes it possible to frame and answer queries based on the minimal common data elements in experimental protocols. This facilitates finding specific protocols; if the owner of the protocol chooses not to expose the full content, as it is the case of publishers and/or laboratories, SIRO may be exposed without compromising the full content of the document. For instance, queries such as "retrieve protocols that use samples from the rodent order" or "retrieve protocols that use Nucleic acid purification kits" are executed using information that is also part of the SIRO model. Retrieving information related to steps, procedures, and recipes is only possible if the protocol is public, e.g. open access. In our case, CIAT facilitated some protocols for which only SIRO elements could be exposed; steps, alert messages and troubleshooting were considered as sensible information that should not be publically available.

\section{Evaluating the SIRO model}

For evaluating SIRO we extracted and populated the SIRO model with the RDF dataset that we used for the evaluation of the SP ontology. As the SIRO model does not expose the whole content of the protocol we also added five unpublished, private, protocols to the dataset. In total, for this evaluation we have 15 protocols in the SPARQL endpoint ${ }^{4}$. For those queries involving instances of SIRO, we could satisfactorily retrieve the information required by the competency questions. Moreover, as SIRO complements bibliographic metadata information, the wealth of queries can be expanded. For instance:

- Retrieve the protocols and the list of reagents for documents authored by Yoshimi Umemura. 
Table 4 Queries making use of external resources. Queries are available at https://smartprotocols.github.io/queries/

\begin{tabular}{|c|c|c|c|c|}
\hline $\begin{array}{l}\text { Competency } \\
\text { question }\end{array}$ & $\begin{array}{l}\text { Was the question } \\
\text { answered? }\end{array}$ & $\begin{array}{l}\text { Other Information } \\
\text { Resources }\end{array}$ & SPARQL & Comment \\
\hline $\begin{array}{l}\text { Retrieve all the } \\
\text { protocols that } \\
\text { use mouse as a } \\
\text { sample }\end{array}$ & $\begin{array}{l}\text { Yes. Could there be a short } \\
\text { description about the } \\
\text { organism and also, mouse } \\
\text { is too specific, I may also } \\
\text { be interested in rats and } \\
\text { other rodents. }\end{array}$ & $\begin{array}{l}\text { The DBPEDIA property } \\
\text { dbo:order of includes } \\
\text { individuals that belong } \\
\text { to the order rodents, } \\
\text { e.g. rats, hamsters, } \\
\text { squirrels, etc. DBPEDIA } \\
\text { also has dbo:abstract, } \\
\text { this property allows us to } \\
\text { retrieve information about } \\
\text { rodents. }\end{array}$ & $\begin{array}{l}\text { Query\#1. Retrieve all the } \\
\text { protocols with samples } \\
\text { that belong to the Rodent } \\
\text { order and also retrieve } \\
\text { information for these } \\
\text { samples }\end{array}$ & $\begin{array}{l}\text { Additional } \\
\text { information } \\
\text { was useful } \\
\text { but basic }\end{array}$ \\
\hline $\begin{array}{l}\text { Retrieve all the } \\
\text { reagents used in } \\
\text { the protocols }\end{array}$ & $\begin{array}{l}\text { Yes. It is also useful to } \\
\text { know where to buy these } \\
\text { products. }\end{array}$ & $\begin{array}{l}\text { PubChem has a list of } \\
\text { vendors for some } \\
\text { reagents. For instance, } \\
\text { for sodium chloride it has } \\
\text { more than ten vendors. } \\
\text { Also, we are resolving the } \\
\text { entities against the } \\
\text { websites of the } \\
\text { manufacturers. }\end{array}$ & $\begin{array}{l}\text { Query \#4.Retrieve all the } \\
\text { reagents along with the } \\
\text { different web sites to buy } \\
\text { them and all the } \\
\text { different manufacturers } \\
\text { registered for every } \\
\text { reagent }\end{array}$ & $\begin{array}{l}\text { Additional } \\
\text { information } \\
\text { was useful }\end{array}$ \\
\hline $\begin{array}{l}\text { Retrieve the } \\
\text { protocols in } \\
\text { which } \\
\text { Bromophenol } \\
\text { blue is used }\end{array}$ & $\begin{array}{l}\text { Yes. Could the } \\
\text { applications for the } \\
\text { reagent be included in the } \\
\text { answer? }\end{array}$ & $\begin{array}{l}\text { ChEBI is an external } \\
\text { resource that has the } \\
\text { applications for some } \\
\text { reagents. }\end{array}$ & $\begin{array}{l}\text { Query \#23 Retrieve the } \\
\text { protocols in which } \\
\text { Bromophenol blue is used } \\
\text { and tell me about the } \\
\text { application of } \\
\text { Bromophenol blue }\end{array}$ & $\begin{array}{l}\text { Additional } \\
\text { information } \\
\text { was useful }\end{array}$ \\
\hline $\begin{array}{l}\text { Retrieve the steps } \\
\text { that have } \\
\text { CAUTIONS as } \\
\text { alert messages } \\
\text { from the protocol } \\
\text { " } \mathrm{X}^{\text {" }}\end{array}$ & $\begin{array}{l}\text { Yes. I would also like to } \\
\text { have the diseases caused } \\
\text { by this reagent }\end{array}$ & $\begin{array}{l}\text { In this case we are } \\
\text { making use of } \\
\text { Bioportal and SNOMED } \\
\text { (causative_agent_of). }\end{array}$ & $\begin{array}{l}\text { Query \#14. Retrieve all the } \\
\text { diseases caused by the } \\
\text { reagents in the protocol } \\
\text { "Extraction of total RNA } \\
\text { from fresh/frozen tissue } \\
(\text { FT)" }\end{array}$ & $\begin{array}{l}\text { Additional } \\
\text { information } \\
\text { was useful }\end{array}$ \\
\hline
\end{tabular}

- Retrieve the protocols authored by Yoshimi Umemura and Beata Dedicova using rice leaves as sample.

- Retrieve the common reagents across the protocols "[Bio101] Subcutaneous Injection of Tumor Cells" and "Scratch Wound Healing Assay".

\section{Discussion SMART protocols ontology}

We propose the SP ontology to represent experimental protocols. It reuses the metadata structure, as well as some classes and properties, from OBI. It also builds upon experiences such as the BioAssay Ontology (BAO), The

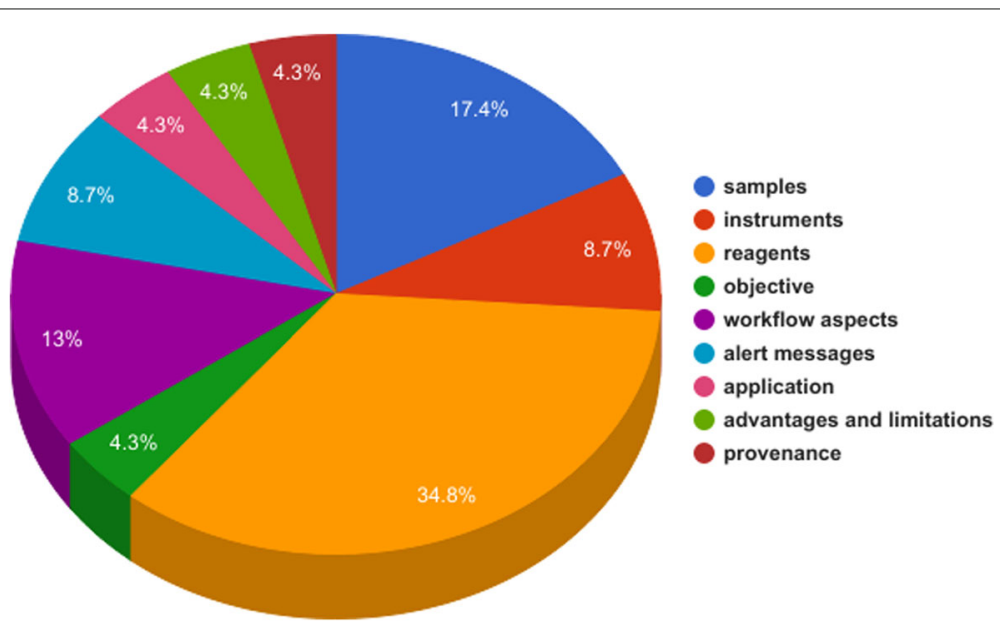

Fig. 4 Distribution of SIRO elements 


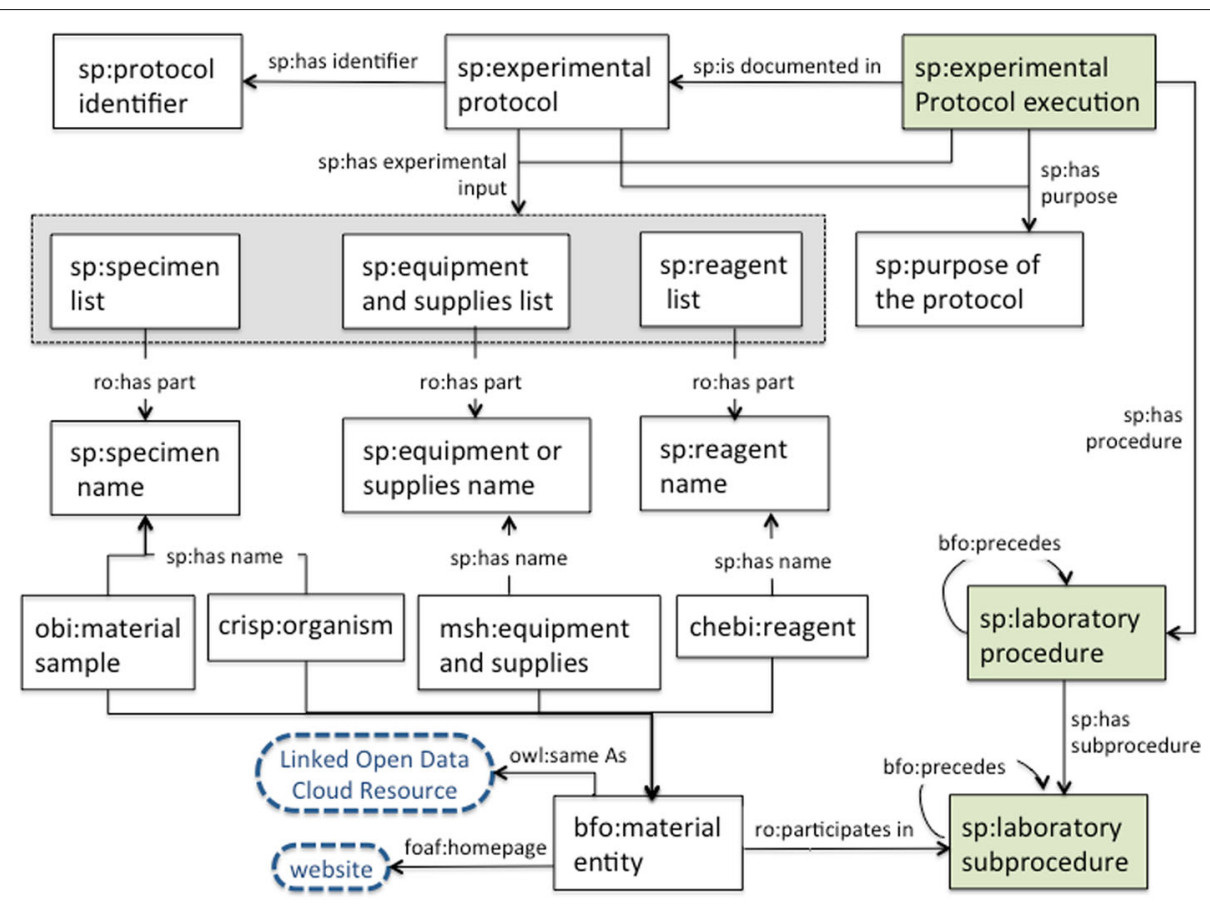

Fig. 5 The SIRO model

Experimental Factor Ontology (EFO), eagle-i resource ontology (ERO) and also the EXACT ontology. The SP Ontology also considers reporting structures such as ARRIVE, BRIDG as well as those from BioSharing. For representing "instruments", "reagents/chemical compounds", "organisms" and "sample/specimen" we reuse, amongst others, NCBI taxonomy, Cell Line Ontology (CLO) and Chemical Entities of Biological Interest (ChEBI). Our results indicate that the SP ontology makes it possible to represent all the data elements in the experimental protocols that we have analyzed.

\section{Modularization of the SP ontology}

Modularization, as it has been implemented in SP, facilitates specializing the ontology with more precise formalisms. For instance, reagents, instruments and experimental procedures (actions), may be instantiated based on the activities carried out by a particular laboratory. We have two main modules in our ontology, the SP-Document and the SP-Workflow modules. The document module address issues related to archiving and representing the narrative. The workflow module aims to deliver a reusable executable object. In this way we make it possible for protocols to "be born semantics". To "be born semantics" delivers a self-describing workflow embedded within a document from the onset. As a document, it is easily managed and understood by humans. As a self-describing workflow embedded within a document it is easily processed by machines. Our representation has some limitations with respect to machine processability; for instance, it is not suitable for robots to interpret it.

The document module facilitates archiving; publishers and laboratories can extend it depending on their use cases. The workflow module delivers an extensible representation describing the sequence of activities in an experimental protocol. Actions, as presented by [11], are important descriptors for biomedical protocols. However, in order for actions to be meaningful, attributes such as measurement units and material entities (e.g. sample, instrument, reagents, personnel involved) are also necessary. Our workflow representation makes it possible to link procedures and subprocedures to reagents, instruments, samples, recipes, hints, alert messages, etc. This is particularly useful because procedures and subprocedures can easily be reused and adapted; also, it allows researchers to retrieve very specific information and aggregate other data elements as it is needed. Formalizing workflows has an extensive history in Computer Science; not only in planning but also in execution as in Process Life-cycle Management and Computer Assisted Design/Computer Assisted Manufacturing. The SP-workflow module helps to formalize the workflow implicit in protocols; our workflow specification has some limitations. For instance, loops, conditionals and other workflow constructs are currently being formalized as new use cases are identified. Our workflow constructs are easily extensible; we are also evaluating formal workflow languages for processes and adapting these to the 
Table 5 SIRO elements

\begin{tabular}{|c|c|c|}
\hline \multirow[t]{6}{*}{ Sample } & Whole organism & $\begin{array}{l}\text { Scientific name: Arabidopsis } \\
\text { thaliana, Oriza sativa, mangifera } \\
\text { indica, Mus musculus. }\end{array}$ \\
\hline & & $\begin{array}{l}\text { Common names: Mousear Cress, } \\
\text { rice, mango, mouse. }\end{array}$ \\
\hline & Anatomical part & $\begin{array}{l}\text { Leaf, stem, cells, tissues, } \\
\text { membranes, organs, skeletal } \\
\text { system, muscular system, } \\
\text { nervous system, reproductive } \\
\text { system, cardiovascular system, etc. }\end{array}$ \\
\hline & Biomolecules & $\begin{array}{l}\text { Nucleic acids: Deoxyribonucleic acid } \\
\text { (DNA) and ribonucleic acid (RNA). }\end{array}$ \\
\hline & & $\begin{array}{l}\text { Proteins: enzymes, structural or } \\
\text { support proteins (keratin, elastin, } \\
\text { collagen), antibodies, hormones, etc. }\end{array}$ \\
\hline & Body fluids & $\begin{array}{l}\text { Blood serum, saliva, semen, amniotic } \\
\text { fluid, cerebrospinal fluid, gastric } \\
\text { acid, etc. }\end{array}$ \\
\hline \multirow[t]{5}{*}{ Instrument } & $\begin{array}{l}\text { High-throughput } \\
\text { equipment }\end{array}$ & $\begin{array}{l}\text { Liquid Handling Platforms, Real-Time } \\
\text { PCR Detection System, Microplate } \\
\text { Reader, etc. }\end{array}$ \\
\hline & Instruments & $\begin{array}{l}\text { Goggles, Bunsen burner, spot plate, } \\
\text { pipet, forceps, test tube rack, mortar } \\
\text { and pestle, etc. }\end{array}$ \\
\hline & Laboratory glassware & $\begin{array}{l}\text { Beaker, Erlenmeyer flask, graduated } \\
\text { cylinder, volumetric flask, etc. }\end{array}$ \\
\hline & Standard equipment & $\begin{array}{l}\text { Balances, shakers, centrifuges, } \\
\text { refrigerators, incubators, } \\
\text { thermocyclers, fume hood, etc. }\end{array}$ \\
\hline & Consumables & $\begin{array}{l}\text { Weighing dishes, pipette tips, gloves, } \\
\text { syringes, petri dishes, test tubes, } \\
\text { micro centrifuge tubes, glass slides, } \\
\text { filter paper, etc. }\end{array}$ \\
\hline \multirow[t]{3}{*}{ Reagents } & $\begin{array}{l}\text { Chemical } \\
\text { compound/Substance }\end{array}$ & $\begin{array}{l}\text { Glucose, ethanol, glycerol, chloroform, } \\
\text { acetic acid, isopropyl alcohol, etc. }\end{array}$ \\
\hline & Solutions/buffers & $\begin{array}{l}\text { 70\% ethanol, 10X PCR buffer, } \\
\text { phenol:chloroform:isoamyl } \\
\text { alcohol, etc. }\end{array}$ \\
\hline & Cell culture media & $\begin{array}{l}\text { Nutrient media, minimal media, } \\
\text { selective media, differential media, etc. }\end{array}$ \\
\hline Objective & Part of discourse & $\begin{array}{l}\text { Here we present a detailed protocol } \\
\text { for Smart-seq } 2 \text { that allows the } \\
\text { generation of full-length cDNA and } \\
\text { sequencing libraries by using } \\
\text { standard reagents }\end{array}$ \\
\hline
\end{tabular}

biomedical scenario. Overcoming the limitations in the description of the workflow will make it possible to have an accurate representation of the protocol as an executable object for machines to fully process -including robots. The workflow nature implicit in experimental protocols should also be intelligible and manageable by humans; we are currently exposing the protocols in a format, RDF, that machines can understand for web purposes, e.g. discovery, interoperability.

\section{Limitations}

Describing samples was particularly difficult because attributes like strain, line or genotype, developmental stage, organism part, growth conditions, age, gender, pretreatment of the sample and volume/mass of sample, etc, are important depending on the experiment and the type of sample. Reagents and instruments were easier to describe as they only require the commercial name, manufacturer and identification number. However, linking reagents and instruments to other information resources is not as simple. Manufacturers don't always offer Application Programing Interfaces (APIs) that make it possible to resolve these entities against their websites. For our experiment we had to scrape these websites in order to build the links. Furthermore, they don't always use controlled vocabularies, common identifiers or describe chemicals in the same way; this made it difficult to search across their catalogs. Sigma-Aldrich and PubChem link to each other and PubChem has links to several manufacturers and vendors, this was deemed useful by domain experts. Linking was not initially considered by domain experts in their early competency questions; however, when they saw the answers for their queries, their expectation for linking data grew. In order to meet this demand, we re-formulated the queries by adding some external resources. This was received with satisfaction by domain experts; however, the expectation for more data continued growing. The use of external data sources was problem dependent, so were the external data sources to use.

\section{The SIRO model, application of the ontology}

The SIRO model for minimal information breaks down the protocol in key elements that we have found to be common across our corpus of experimental protocols: $i$ ) Sample/ Specimen (S), ii) Instruments (I), iii) Reagents (R) and iv) Objective (O). Exposing SIRO makes it possible for laboratories and publishers to present key elements that frame questions often asked by researchers when searching for experimental protocols. SIRO was tested and results were satisfactory. External sources of information, e.g. vendor information from PubChem, can also be used to enrich SIRO elements. By extending the bibliographic metadata, SIRO is also extending the wealth of queries being supported; it provides specific information that is relevant to the description of the protocol.

\section{Conclusions}

Experimental protocols are central to reproducibility and they are widely used in experimental laboratories. Our 
ontology and minimal information model have been validated with domain experts; our evaluations indicate that the SP ontology can represent experimental workflows and also that retrieving specific information from protocols represented with the SP ontology is possible. Both, the ontology and the SIRO model are easily adaptable. Experimental protocols describe step by step "how to do or how to execute" an experimental procedure. In our conceptualization experimental protocols have a document and a workflow component; as workflows embedded within documents, the experimental protocols should have complete information that allows anybody to recreate an experiment.

Our approach facilitates the generation of a selfdescribing document. It makes it possible to present meaningful information of experimental protocols without compromising the content. More importantly, it makes it possible to anchor information retrieval within a context that is meaningful for experimental researchers, e.g. reagents, samples and instruments participating in subprocedures. Queries such as "What DNA extraction protocol is used on rice samples?", "what amount of leaf tissue to use?" are common for experimental researchers; answering these is possible with the SP ontology. In laboratory settings experimental protocols are usually managed just like any other document. However, these are plans for the execution of experiments; resources are allocated based on specifics described in the workflows of experimental protocols. The SMART Protocols approach generates a computable document that may interoperate with, for instance, inventories or Laboratory Information Management Systems (LIMS). Thus making it easier for researchers to plan according to available resources.

Harmonizing efforts such as EXACT, OBI, STAR [10], BRIDG and SMART Protocols ontology is important because without a clear semantics, reporting structure and a minimal information model for experimental protocols these will remain highly idiosyncratic. Moreover, without such consensus the experimental record will remain highly fragmented and therefore not easily processable by machines or reproducible by humans. Efforts such as the Resource Identification Initiative (RRId) $[32,53]$ and identifiers.org $[54,55]$ are central in the preservation of the experimental record; it is important that these efforts start to address reagents and instruments more broadly as these resources don't always have identifiers. Being able to review the data makes it possible to evaluate whether the analysis and conclusions drawn are accurate. However, it does little to validate the quality and accuracy of the data itself. The data must be available, so does the experimental protocol detailing the methodology followed to derive the data. Journals and founders are now asking for datasets to be publicly available; there have been several efforts addressing the problem of data repositories; if data must be public and available, shouldn't researchers be held to the same principle when it comes to methodologies? Openness and reproducibility are not only related to data availability; when replicating research, being able to follow the steps leading to the production of data is equally important.

The SP ontology is a digital object that follows the FAIR Principles [56]. Our ontology is findable; it is registered at Bioportal ${ }^{5}$, it is also available in github ${ }^{6}$ and the vocab.linkeddata.es ${ }^{7}$. The ontology is documented to facilitate the reusability; classes and object properties are documented with annotation properties imported from the OBI Minimal metadata. Reusing the ontology is easy as it has "preferred terms", "definitions", "definition sources", "example of use", "alternative terms", etc; this makes it easier for others to know the context of the terminology as well as the suitability for addressing other use cases. The SP ontology was developed in OWL-DL and it is licensed under a Creative Commons Attribution 4.0 International License; in this sense SP ontology is interoperable and accessible.

\section{Endnotes}

${ }^{1}$ http://smartprotocols.linkeddata.es/sparql

${ }^{2}$ https://smartprotocols.github.io/queries/

${ }^{3}$ https://smartprotocols.github.io/annex/

${ }^{4}$ https://smartprotocols.github.io/protocolsrdf/

${ }^{5}$ http://bioportal.bioontology.org/ontologies/SP

${ }^{6}$ https://smartprotocols.github.io/

${ }^{7}$ http://vocab.linkeddata.es/SMARTProtocols/

\section{Abbreviations}

BAO: The BioAssay ontology; BFO: The basic formal ontology; ChEBI: Chemical entities of biological interest; CIAT: Center for tropical agriculture; DAKA:

Domain analysis and knowledge acquisition; DNA: Deoxyribonucleic acid; EFO; The experimental factor ontology; ERO: Eagle-i resource ontology; EXACT: The ontology of experimental actions; FT: Fresh tissue or frozen tissue; IAO: The information artifact ontology; LIMS: Laboratory information management; LISA: Linguistic and semantic analysis; MI: Minimal Information; NCBI taxonomy: The national center for biotechnology information taxonomy; NeOn methodology: Methodology for building ontology networks; OBI: The Ontology for biomedical investigations; OOPS: The ontology pitfall scanner; OWL-DL: Web ontology language based on description logic; PCR: The polymerase chain reaction; PICO model: Patient intervention comparison outcome; RNA: Ribonucleic acid; RO: The ontology of relations; SIRO model: Sample instrument reagent objective model; SMART Protocols: semantic representation for protocols; SP ontology: SMART protocols ontology; SP-Document: The document module of SMART protocols ontology; SP-Workflow: The workflow module of SMART protocols ontology; SPARQL: SPARQL protocol and RDF query language

\section{Acknowledgements}

We thank everyone at CIAT; in particular we want to express our gratitude to Joe Tohme, Adriana Bohorquez, Constanza Quintero, Alba Lucia Chavez, Maria Eugenia Recio, Ivan Lozano, Wilmer Cuellar, Viviana Ortiz, Carlos Andres Cotes, Victoria Arredondo and Soroush Parsa for their participation in workshops and discussions. We also want to thank Melissa Carrion for her useful comments and proof-reading. Finally, we would like to thank the anonymous reviewers for their valuable comments and suggestions to improve the manuscript. 


\section{Funding}

This work has been funded by the EU project Drinventor FP7-ICT-2013.8.1, as well as by the KOPAR project, H2020-MSCA-IF-2014, Grant Agreement nr: 655009; and by the Spanish MINECO and co-financed by the BEI and FEDER through 4V project Ref: TIN2013-46238-C4-2-R.

\section{Availability of data and materials}

The ontology, corpus of protocols, annexes cited in the document, protocols as RDF and competency questions are available at https://smartprotocols. github.io/.

\section{Data availability}

All data generated or analyzed during this study are included in this published article. everything is available at https://smartprotocols.github.io/.

\section{Authors' contributions}

OG developed the ontology and the minimal information model. OG and AG provided biological expertise. OG and FL implemented the representation of protocols as RDF. AG and OC supervised the work and revised the manuscript. All authors read and approved the final manuscript.

\section{Ethics approval and consent to participate}

Not applicable.

\section{Consent for publication}

Not applicable.

\section{Competing interests}

The authors declare that they have no competing interests.

\section{Publisher's Note}

Springer Nature remains neutral with regard to jurisdictional claims in published maps and institutional affiliations.

Received: 2 June 2016 Accepted: 15 October 2017

Published online: 13 November 2017

\section{References}

1. Giraldo O, Garcia A, Oscar C. SMART Protocols: SeMAntic RepresenTation for Experimental Protocols. In: 4th Workshop on Linked Science 2014Making Sense Out of Data (LISC2014). Riva del Garda, Trentino, Italy; 2014.

2. Acevedo LG, Iniguez AL, Holster HL, Zhang X, Green R, Farnham PJ. Genome-scale ChIP-chip analysis using 10,000 human cells. Biotechniques. 2007;43(6):791-7.

3. Kilkenny C, Browne WJ, Cuthill IC, Emerson M, Altman DG. Improving bioscience research reporting: the arrive guidelines for reporting animal research. PLoS Biol. 2010;8(6):1000412.

4. Nature Protocols Guide to Authors. http://www.nature.com/nprot/info/ gta.html. Accessed 7 May 2016.

5. Plant Methods - BioMed Central, Submission Guidelines. http:// plantmethods.biomedcentral.com/submission-guidelines/preparingyour-manuscript/methodology. Accessed 7 May 2016.

6. Cold Spring Harbor Protocols, Instructions For authors. http://cshlpress. com/cshprotocols/. Accessed 7 May 2016.

7. Rocca-Serra P, Sansone SA, Brand M. Release Candidate 1, ISA-TAB v1.0 Specification Document, Version 24th; 2008, p. 36. http://isatab. sourceforge.net/docs/ISA-TAB release-candidate-1_v1.0_24nov08.pdf.

8. Biomedical Research Integrated Domain Group. https://bridgmodel.nci. nih.gov/. Accessed 10 Feb 2017.

9. Taylor CF, Field D, Sansone SA, Aerts J, Apweiler R, Ashburner M, Ball CA, Binz PA, Bogue M, Booth T, Brazma A, Brinkman RR, Michael Clark A, Deutsch EW, Fiehn O, Fostel J, Ghazal P, Gibson F, Gray T, Grimes G, Hancock JM, Hardy NW, Hermjakob H, Julian RK, Kane M, Kettner C, Kinsinger C, Kolker E, Kuiper M, Novere NL, Leebens-Mack J, Lewis SE, Lord P, Mallon AM, Marthandan N, Masuya H, McNally R, Mehrle A, Morrison N, Orchard S, Quackenbush J, Reecy JM, Robertson DG, Rocca-Serra P, Rodriguez H, Rosenfelder H, Santoyo-Lopez J, Scheuermann RH, Schober D, Smith B, Snape J, Stoeckert CJ, Tipton K, Sterk P, Untergasser A, Vandesompele J, Wiemann S. Promoting coherent minimum reporting guidelines for biological and biomedical investigations: the MIBBI project. Nat Biotechnol. 2008;26(8):889-96.
10. Marcus E. A STAR Is Born. Cell. 2016;166(5):1059-60.

11. Soldatova LN, Aubrey W, King RD, Clare A. The EXACT description of biomedical protocols. Bioinformatics. 2008;24(13):295-303.

12. Soldatova $L N$, Nadis $D$, King RD, Basu PS, Haddi E, Baumlé $V$, Saunders NJ, Marwan W, Rudkin BB. EXACT2: the semantics of biomedical protocols. BMC Bioinforma. 2014;15(Suppl 14):5.

13. Abeyruwan $\mathrm{S}$, Vempati UD, Küçük-McGinty H, Visser U, Koleti A, Mir A, Sakurai K, Chung C, Bittker JA, Clemons PA, Brudz S, Siripala A, Morales AJ, Romacker M, Twomey D, Bureeva S, Lemmon V, Schürer SC. Evolving BioAssay ontology (BAO): modularization, integration and applications. J Biomed Semant. 2014;5(Suppl 1 Proceedings of the Bio-Ontologies Spec Interest G):5.

14. Torniai C, Brush M, Vasilevsky N, Segerdell E, Wilson M, Johnson $T$, Corday K, Shaffer C, Haendel M. Developing an application ontology for biomedical resource annotation and retrieval: Challenges and lessons learned. In: Proceedings of the second international conference on biomedical ontology. Buffalo; 2011. p. 101-8. [http://icbo.buffalo.edu/ ICBO-2011_Proceedings.pdf].

15. Hastings J, de Matos $P$, Dekker A, Ennis M, Harsha B, Kale N, Muthukrishnan V, Owen G, Turner S, Williams M, Steinbeck C. The ChEBI reference database and ontology for biologically relevant chemistry: enhancements for 2013. Nucleic Acids Res. 2013;41:456-63.

16. Federhen S. Type material in the NCBI Taxonomy Database. Nucleic Acids Res. 2015;43:1086-98.

17. Benson DA, Karsch-Mizrachi I, Lipman DJ, Ostell J, Sayers EW. GenBank. Nucleic Acids Res. 2009;37(Database):26-31.

18. Sayers EW, Barrett T, Benson DA, Bryant SH, Canese K, Chetvernin V, Church DM, DiCuccio M, Edgar R, Federhen S, Feolo M, Geer LY, Helmberg W, Kapustin Y, Landsman D, Lipman DJ, Madden TL, Maglott DR, Miller V, Mizrachi I, Ostell J, Pruitt KD, Schuler GD, Sequeira E, Sherry ST, Shumway M, Sirotkin K, Souvorov A, Starchenko G, Tatusova TA, Wagner L, Yaschenko E, Ye J. Database resources of the National Center for Biotechnology Information. Nucleic Acids Res. 2009:37(Database):5-15.

19. Bandrowski $A$, Brinkman $R$, Brochhausen $M$, Brush $M H$, Bug $B$, Chibucos MC, Clancy K, Courtot M, Derom D, Dumontier M, Fan L, Fostel J, Fragoso G, Gibson F, Gonzalez-Beltran A, Haendel MA, He Y, Heiskanen M, Hernandez-Boussard T, Jensen M, Lin Y, Lister AL, Lord P, Malone J, Manduchi E, McGee M, Morrison N, Overton JA, Parkinson H, Peters B, Rocca-Serra P, Ruttenberg A, Sansone SA, Scheuermann RH, Schober D, Smith B, Soldatova LN, Stoeckert CJ, Taylor CF, Torniai C Turner JA, Vita R, Whetzel PL, Zheng J. The Ontology for Biomedical Investigations. PLOS ONE. 2016;11(4):0154556.

20. Malone J, Holloway E, Adamusiak T, Kapushesky M, Zheng J, Kolesnikov N, Zhukova A, Brazma A, Parkinson H. Modeling sample variables with an Experimental Factor Ontology. Bioinformatics. 2010;26(8):1112-8.

21. Sarntivijai $S$, Lin $Y$, Xiang $Z$, Meehan TF, Diehl AD, Vempati UD, Schürer SC, Pang C, Malone J, Parkinson H, Liu Y, Takatsuki T, Saijo K, Masuya H, Nakamura Y, Brush MH, Haendel MA, Zheng J, Stoeckert CJ, Peters B, Mungall CJ, Carey TE, States DJ, Athey BD, He Y. Clo: The cell line ontology. J Biomed Semant. 2014;5:37-7.

22. Sarntivijai S, Xiang Z, Meehan T, Diehl A, Vempati U, Schurer S, Pang C, Malone J, Parkinson H, Athey B, He Y. Cell line ontology: Redesigning the cell line knowledgebase to aid integrative translational informatics. Neoplasia. 2011;833:25-32.

23. Information Artifact Ontology (IAO). https://github.com/informationartifact-ontology//AO/. Accessed 7 May 2016.

24. Coggan JM. Evidence-based practice for information professionals: A handbook. J Med Lib Assoc. 2004;92(4):503-3.

25. Linton KM, Hey Y, Dibben S, Miller CJ, Freemont AJ, Radford JA, Pepper SD. Extraction of total RNA from fresh/frozen tissue (FT). Int J Life Sci Methods. 2010;53.

26. SMART Protocols Project in Github. https://github.com/oxgiraldo/ SMART-Protocols. Accessed 7 May 2016.

27. Suárez-Fiqueroa MC, Gómez-Pérez A, Fernández-López M. The NeOn Methodology for Ontology Engineering. Berlin: Springer; 2012, pp. 9-34. http://oa.upm.es/21469/.

28. Castro A, Rocca-Serra P, Stevens R, Taylor C, Nashar K, Ragan MA Sansone SA. The use of concept maps during knowledge elicitation in ontology development processes - the nutrigenomics use case. BMC Bioinforma. 2006;7(1):267. 
29. Smith B, Ashburner M, Rosse C, Bard J, Bug W, Ceusters W, Goldberg LJ, Eilbeck K, Ireland A, Mungall CJ, Leontis N, Rocca-Serra P, Ruttenberg A, Sansone SA, Scheuermann RH, Shah N, Whetzel PL, Lewis S. The obo foundry: coordinated evolution of ontologies to support biomedical data integration. Nat Biotech. 2007;25(11):1251-5.

30. Whetzel PL, Noy NF, Shah NH, Alexander PR, Nyulas C, Tudorache T, Musen MA. BioPortal: enhanced functionality via new Web services from the National Center for Biomedical Ontology to access and use ontologies in software applications. Nucleic Acids Res. 2011;39(Web Server issue):541-5.

31. Xiang Z, Mungall C, Ruttenberg A, He Y. Ontobee: A Linked Data Server and Browser for Ontology Terms. In: Proceedings of the 2nd International Conference on Biomedical Ontologies (ICBO). Buffalo; 2011. p. 279-281. http://ceur-ws.org/Vol-833/paper48.pdf. Accessed 26 Oct 2017.

32. Publishing in the 21 st century: Minimal (really) data standards | FORCE11. https://www.force11.org/node/4145. Accessed 9 Feb 2017.

33. Zimmermann $P$, Schildknecht $B$, Craigon D, Garcia-Hernandez $M$, Gruissem W, May S, Mukherjee G, Parkinson H, Rhee S, Wagner U, Hennig L. MIAME/Plant - adding value to plant microarrray experiments. Plant Methods. 2006;2(1):1.

34. Bustin SA, Benes V, Garson JA, Hellemans J, Huggett J, Kubista M, Mueller R, Nolan T, Pfaffl MW, Shipley GL, Vandesompele J, Wittwer CT. The MIQE Guidelines: Minimum Information for Publication of Quantitative Real-Time PCR Experiments. Clin Chem. 2009;55(4):611-22.

35. Gibson F, Anderson L, Babnigg G, Baker M, Berth M, Binz PA, Borthwick A, Cash P, Day BW, Friedman DB, Garland D, Gutstein HB, Hoogland C, Jones NA, Khan A, Klose J, Lamond Al, Lemkin PF, Lilley KS, Minden J, Morris NJ, Paton NW, Pisano MR, Prime JE, Rabilloud T, Stead DA, Taylor CF, Voshol H, Wipat A, Jones AR. Guidelines for reporting the use of gel electrophoresis in proteomics. Nat Biotechnol. 2008;26(8):863-4.

36. Protégé. http://protege.stanford.edu/. Accessed 11 Apr 2017.

37. International Center for Tropical Agriculture (CIAT). https://ciat.cgiar.org/. Accessed 20 May 2016.

38. Bachlava E, Taylor CA, Tang S, Bowers JE, Mandel JR, Burke JM, Knapp SJ. SNP Discovery and Development of a High-Density Genotyping Array for Sunflower. PLOS ONE. 2012;7(1):29814.

39. Wang L, Si Y, Dedow LK, Shao Y, Liu P, Brutnell TP. A Low-Cost Library Construction Protocol and Data Analysis Pipeline for Illumina-Based Strand-Specific Multiplex RNA-Seq. PLoS ONE. 2011;6(10):26426.

40. Hasan S, Prakash J, Vashishtha A, Sharma A, Srivastava K, Sagar F, Khan N, Dwivedi K, Jain P, Shukla S, et al. Optimization of dna extraction from seeds and leaf tissues of chrysanthemum (chrysanthemum indicum) for polymerase chain reaction. Bioinformation. 2012;8(5):225.

41. OWLViz. http://protegewiki.stanford.edu/wiki/OWLViz. Accessed 30 May 2016.

42. OOPS! (OntOlogy Pitfall Scanner!). http://oops.linkeddata.es/. Accessed 30 May 2016.

43. Poveda-Villalón M, Suárez-Figueroa M, Gómez-Pérez A. Validating Ontologies with OOPS! In: ten Teije A, et al., editors. Knowledge Engineering and Knowledge Management. Berlin: Springer; 2012. p. 267-81.

44. Smith B, Ceusters W, Klagges B, Köhler J, Kumar A, Lomax J, Mungall C, Neuhaus $F$, Rector $A L$, Rosse $C$. Relations in biomedical ontologies. Genome Biol. 2005;6(5):46.

45. OBI Minimal metadata - OBI Ontology. http://obi.sourceforge.net/ ontologylnformation/MinimalMetadata.html. Accessed 9 Feb 2017.

46. Documentation of SMART Protocols Ontology: Document Module. http://vocab.linkeddata.es/SMARTProtocols/myDocumentation_SPdoc_ 18Abril2017/index_SPdoc_V4.0.html. Accessed 26 Oct 2017.

47. Documentation of SMART Protocols Ontology: Workflow Module. http://vocab.linkeddata.es/SMARTProtocols/myDocumentation_SPwf_ 19Abril2017/index_SPwf_V4.0.html. Accessed 26 Oct 2017.

48. Relations Ontology. http://obofoundry.org/ontology/ro.html. Accessed 13 Apr 2017.

49. Hastings J, de Matos P, Dekker A, Ennis M, Harsha B, Kale N, Muthukrishnan V, Owen G, Turner S, Williams M, Steinbeck C. The ChEBI reference database and ontology for biologically relevant chemistry: enhancements for 2013. Nucleic Acids Res. 2013;41(D1):456-63.

50. Kim S, Thiessen PA, Bolton EE, Chen J, Fu G, Gindulyte A, Han L, He J, He S, Shoemaker BA, Wang J, Yu B, Zhang J, Bryant SH. PubChem
Substance and Compound databases. Nucleic Acids Res. 2016:44(D1): 1202-13.

51. Auer S, Bizer C, Kobilarov G, Lehmann J, Cyganiak R, Ives Z. Dbpedia: A nucleus for a web of open data. Semant Web. 2007;4825:722-35.

52. Consortium TU. Uniprot: the universal protein knowledgebase. Nucleic Acids Res. 2017;45(D1):158.

53. Bandrowski A, Brush M, Grethe JS, Haendel MA, Kennedy DN, Hill S, Hof PR, Martone ME, Pols M, Tan S, Washington N, Zudilova-Seinstra E, Vasilevsky N, Resource Identification Initiative Members are listed here: https://www.force11.org/node/4463/members. The Resource Identification Initiative: A cultural shift in publishing. F1000Research. 2015;4:134.

54. Juty N, Le Novere N, Laibe C. Identifiers.org and MIRIAM Registry: Community resources to provide persistent identification. Nucleic Acids Res. 2012;40(D1):580-6.

55. Identifiers.org. http://identifiers.org/. Accessed 10 Feb 2017.

56. Wilkinson MD, Dumontier M, Aalbersberg IJ, Appleton G, Axton M, Baak A, Blomberg N, Boiten JW, da Silva Santos LB, Bourne PE, Bouwman J, Brookes AJ, Clark T, Crosas M, Dillo I, Dumon O, Edmunds S, Evelo CT, Finkers R, Gonzalez-Beltran A, Gray AJ, Groth P, Goble C, Grethe JS, Heringa J, 't Hoen PA, Hooft R, Kuhn T, Kok R, Kok J, Lusher SJ, Martone ME, Mons A, Packer AL, Persson B, Rocca-Serra P, Roos M, van Schaik R, Sansone SA, Schultes E, Sengstag T, Slater T, Strawn G, Swertz MA, Thompson M, van der Lei J, van Mulligen E, Velterop J, Waagmeester A, Wittenburg P, Wolstencroft K, Zhao J, Mons B. The fair guiding principles for scientific data management and stewardship. Sci Data. 2016;3:160018.

\section{Submit your next manuscript to BioMed Central and we will help you at every step:}

- We accept pre-submission inquiries

- Our selector tool helps you to find the most relevant journal

- We provide round the clock customer support

- Convenient online submission

- Thorough peer review

- Inclusion in PubMed and all major indexing services

- Maximum visibility for your research

Submit your manuscript at www.biomedcentral.com/submit
Biomed Central 